Transportation Research Forum

A Quantitative Evaluation of the Nighttime Visual Sign Inspection Method

Author(s): William J. Rasdorf, Joseph E. Hummer, Stephanie C. Vereen, and Hubo Cai

Source: Journal of the Transportation Research Forum, Vol. 44, No. 1 (Spring 2005), pp. 121-139

Published by: Transportation Research Forum

Stable URL: http://www.trforum.org/journal

The Transportation Research Forum, founded in 1958, is an independent, nonprofit organization of transportation professionals who conduct, use, and benefit from research. Its purpose is to provide an impartial meeting ground for carriers, shippers, government officials, consultants, university researchers, suppliers, and others seeking exchange of information and ideas related to both passenger and freight transportation. More information on the Transportation Research Forum can be found on the Web at www.trforum.org. 


\section{A Quantitative Evaluation of the Nighttime Visual Sign Inspection Method}

A research project to determine the appropriate sign inspection and replacement procedure was conducted at North Carolina State University and sponsored by the North Carolina DOT. The purpose was to determine the optimum strategy for sign inspection and replacement under different conditions to respond to the pending retroreflectivity requirements. This paper reports on a spreadsheet tool developed to quantitatively evaluate the effectiveness of different sign inspection and replacement scenarios. The spreadsheet was designed for yellow and red engineer-grade sign sheetings, and takes into account sign vandalism and knock-downs as well as normal sign aging. The spreadsheet provides estimates of the number of signs in place that would not meet the minimum retroreflectivity standard and the cost of the sign inspection and replacement program.

The results from a number of trials of the spreadsheet show that agencies that generally conform to the key assumptions made to build the spreadsheet should consider replacing all signs every seven years, as that insures that no aged signs are in place at a relatively low cost. If total replacement is not possible, an inspection program using retroreflectometers every three years appears very competitive in its effectiveness with a program using typical visual inspection rates each year. The retroreflectometers appear to allow fewer deficient signs, while the typical visual inspection program costs are lower for a given vandalism rate. More conservative visual sign replacement rates do not appear to offer distinct advantages, because typical replacement rates with visual inspections every two or three years allow relatively high numbers of deficient signs to remain on the roads.

\section{by William J. Rasdorf, Joseph E. Hummer, Stephanie C. Vereen and Hubo Cai}

Transportation agencies such as state Departments of Transportation (DOTs) are responsible for managing roads and signs across the United States. One of the concerns of state DOTs is sign inventory and management. Statistics from the National Highway Traffic Safety Administration reveal that in 1999, there were 286,000 crashes because of stop sign issues nationwide (Congressional Information Service, 1999). The Institute of Transportation Engineers (ITE) Traffic Sign Handbook states, "if signing is done improperly, longer driver response times, inappropriate responses, or errors will result, all of which adversely affect safety" (Institute of Transportation Engineers, 1997). A report by the Federal Highway Administration (FHWA) reveals that the risk of dying in a crash at night, when signs are more difficult to see, is nearly three times that of dying in the daytime (U. S. Department of Transportation, 1999). It is imperative that DOTs have effective sign testing and replacement programs to significantly reduce the safety risks to motorists.
One important aspect of sign performance is retroreflectivity, measured by a coefficient of retroreflection. The coefficient of retroreflection (Ra) can be understood as the ratio of the light which the sign reflects to a driver (cd) to the light which illuminates the sign (lx) per unit area $\left(\mathrm{m}^{2}\right)$. The English unit for $\mathrm{Ra}$ is cd/fc/sf (candelas per foot-candle per square foot). Ra has the same value in the metric system. It is often referred to as specific intensity per unit area (SIA).

The effectiveness of retroreflective (light returned to the driver) sign sheeting has not been quantified. Beginning in 1984, the Center for Auto Safety petitioned FHWA to establish standards for retroreflectivity. In 1993, the Department of Transportation Appropriation Act stated that the U. S. Secretary of Transportation should revise the Manual for Uniform Traffic Control Devices (MUTCD) to include "a standard for a minimum level of retroreflectivity that must be maintained for pavement markings and signs, which shall apply to all roads open to public travel" (AASHTO, 2000). FHWA 
formulated two related reports in 1998. One report aimed at "evaluating the applicability and practicality of the minimum-maintained levels of sign retroreflectivity proposed by FHWA and the hand-held retroreflectometer that measures sign retroreflectivity" (McGee and Taori, 1998). The other report aimed at providing explanations and procedures to assist agencies in developing their own sign management systems to meet the minimum retroreflectivity requirements (McGee and Paniati, 1998). Although the 2000 edition of the MUTCD did not include retroreflectivity guidelines, Section 2A.09 of the MUTCD is reserved for their future addition.

New retroreflectivity requirements in the pending standard will present several new issues to state transportation agencies responsible for sign replacement and maintenance. In the case of North Carolina, the state owns and maintains approximately 78,000 miles of roadway. Interstate and primary roads contain approximately 388,000 signs and secondary roads contain approximately 605,000 signs (Kirtley and Rasdorf, 2001; Palmquist and Rasdorf, 2002). The new standard poses serious implementation challenges to the North Carolina DOT. Other state DOTs face similar challenges. When these new standards are finally adopted, both compliance (for the safety and well-being of the public) and proof of compliance (to protect against lawsuits) will be necessary.

To meet the proposed new standard, state DOTs have to develop their own sign inspection and replacement procedures. Current procedures will have to be examined, together with alternatives, to determine the optimum sign inspection and replacement procedure.

A research project to determine the appropriate sign inspection and replacement procedure was conducted at North Carolina State University, sponsored by the North Carolina DOT. The purpose was to determine the optimum strategy for sign inspection and replacement under different conditions to respond to the pending retroreflectivity requirements. This paper reports on some of the findings of this research project. In particular, the paper focuses on a tool developed to investigate the effectiveness of different sign inspection and replacement scenarios.

\section{NIGHTTIME SIGN INSPECTION}

Nighttime observation of signs is currently the most commonly used procedure in the United States to determine whether signs are visually adequate. Generally, in this procedure, a twomember team (a driver and a recorder) will drive a vehicle along a road observing and measuring the conditions of road signs and determining the appropriate actions to be taken such as replacement and repair.

\section{The North Carolina DOT Nighttime Visual Inspection Method}

The North Carolina DOT currently employs a nighttime visual sign inspection method to find failed signs along state-maintained roads. Sign condition is evaluated based on the observers' visual observation without using any retroreflectivity measurement equipment. Most inspectors are experienced. New or temporary employees are always paired with an experienced observer.

The North Carolina DOT inspection method is simply to follow a predetermined driving path and evaluate all signs along this path to determine if they are visually adequate. Roads are driven in both directions. However, on many secondary roads, where signs are sparse, the crew will slow the car and shine a light back on signs facing the opposite direction for evaluation. Visually, signs are evaluated at posted speed limits, using the headlights of the car as the light source. If a sign is determined to be questionable, the crew will get out of the car to take a closer look at the sign sheeting and check the sticker indicating the installation date of the sign to determine the appropriate action to be taken, such as replacement, repair, or cleaning. The recorder uses a state-issued form to inventory signs needing replacement. Signs not needing replacement, but requiring repair or cleaning, are recorded on a separate form.

During the nighttime visual inspections, conditions other than retroreflectivity that affect the visibility of the signs are also evaluated. For example, signs might be placed incorrectly or be obstructed by bushes. These conditions will 
also lead to appropriate actions to be taken so that proper placement and visibility are achieved.

It is noted that employee performance is also an important factor in evaluating sign conditions. The same employee may work in a county for many years, may be very familiar with the roads, and may take great pride in ensuring that the signs in the area for which he/she is responsible are in excellent condition. But some employees may not be as meticulous and new or temporary employees may not feel as great a sense of pride and responsibility for maintaining the signs. This results in a lower work standard and poor sign replacement practices.

\section{Retroreflectivity Measurement}

There are currently two main methods to assess the retroreflectivity of a sign in the field. The first is a visual assessment as is being used by the North Carolina DOT. Visual inspection can be performed at night using a bright light and the human eye. The second method to assess retroreflectivity utilizes retroreflectometers, either hand-held or mobile. Neither of the two methods is completely accurate. The accuracy of visual assessment is questionable because different individuals may have varying visual observations of the same sign, even given the same set of inspection guidelines. The use of hand-held retroreflectometers can be time-consuming, often requiring up to four readings per color. Mobile retroreflectometers are still being developed and improved. "There are currently no traceable methods in the United States to determine the accuracy of retroreflectivity measurements because national calibration standards for retroreflectivity do not exist" (AASHTO, 2000).

Mobile Measurement. The Federal Highway Administration has a prototype van able to measure sign retroreflectivity while moving at normal highway speeds. It is known as SMARTS, or Sign Management and Retroreflectivity Tracking System. The van uses a calibrated strobe lamp, mounted on its top, to bounce light off highway signs. The returned light is measured and processed by computer to account for observation angle. It is then compared to guidelines (Hatzi, 2001).
Vans like this may be a common future method for evaluating signs, but they are not ready for widespread use. Using the FHWA van to measure multiple signs in a row is difficult because a successive sign closer than 200 feet falls beneath the tracking range distance. Also, the software is programmed to look for the brightest signs. This is not ideal because out of a group of signs the one needing replacement is the one missed. When left, right, and overhead signs are located at the same milepost, multiple passes would be required to measure all of them, which could prove to be very costly.

The cost per mile to run the FHWA van is unknown. The initial cost of the SMARTS van and all of the equipment and software was about $\$ 210,000$. This does not include continuous maintenance and upgrades. This van is several years old and to reproduce the same van today would cost much more. Data validating the accuracy and repeatability of measurements recorded in the van are not yet available.

Hand-Held Measurement. Hand-held retroreflectometers are instruments capable of measuring the retroreflectivity of signs, that is, the amount of light reflected back to the driver. They range in weight up to around five pounds, although newer units are lighter. They can be transported easily in the field. Most available units are equipped with rechargeable batteries, some of which are able to be charged in as little as 15 minutes. Currently there are several models available with varying capabilities. The underlying function and principle behind each model is the same. When pointed at, or placed directly against a sign, the instrument emits a beam of light and measures the amount of reflected light returned from the sign's sheeting. The unit can be operated by one person but may sometimes require an extension pole to reach tall signs. The operator must be positioned in front of a sign. Some models have to be placed directly on the sign being evaluated while others operate at ranges of around 50 to 100 feet from the sign.

To maintain accuracy, retroreflectometers are typically calibrated for each sheeting color and type before use. Most models come with a calibration standard with known retroreflectivity levels, which is used to test the instrument at a time interval determined by the manufacturer. 
The reference standard is supplied by the manufacturer in a storage case that is rarely carried to the field where it may be altered by uncontrolled conditions. "Although the use of retroreflectometers is an objective measurement method, it can be expensive and time-consuming. As many as 60 measurements may be needed to evaluate the retroreflectivity of a large sign; frequently a lane of traffic must be closed to do so" (Long, 1997).

Some units are capable of data collection, storage, and download. The storage capacity varies from unit to unit. Stored measurements can later be downloaded to a computer. However, the number of measurements held by the instrument is sometimes limited to a little more than 1,000 for some models. If at least four measurements are taken per sign, then only about 250 signs can be measured before the data will need to be downloaded into a computer. Retroreflectometers cost up to $\$ 9,000$, although this varies among manufacturers and models.

\section{Comparison of Measurement Methods}

The previously-mentioned methods to inspect signs have advantages and disadvantages that are summarized in Table 1. The visual inspection method is efficient compared to the others and it can identify missing and vandalized signs. However, it does not generate numerical data about each sign (signs are evaluated either as currently acceptable or as needing attention such as replacement, repair, or cleaning). Finally, visual inspection is labor- and time-intensive and its reliability and accuracy are uncertain.

Hand-held retroreflectivity measurement units are capable of providing numerical data. However, state DOTs are concerned that measurements with hand-held units would be slow. The accuracy and reliability of these instruments are also questionable. "There can be significant variability among instruments measuring the same object, and the standards do not ensure the accuracy of the instruments" (NCHRP, 2003). Currently, there are no national calibration standards for retroreflectivity, but NCHRP Project 5-16 is dedicated to this task (NCHRP, 2003).

A mobile measurement van would be able to solve many of the problems a hand-held unit cannot, particularly faster measurement. However, vans are not yet ready to be relied on for commercial use. A mobile unit will be expensive regardless of whether it is assembled independently by the state or if a service is contracted to do the work.

In North Carolina, previous studies determined that there are approximately $1,000,000$ in-place signs along state-maintained roads in North Carolina (Kirtley and Rasdorf, 2001; Palmquist and Rasdorf, 2002). The North Carolina DOT does not believe that measuring all these signs using a hand-held unit is realistic because of the time required. According to a Washington State study (Lagergren 1987), a measurement rate of 10 signs per hour for ground mounted warning and stop signs located on the

Table 1: Comparison of Inspection Methods (Numerical vs. Non-numerical Generation)

\begin{tabular}{|c|c|c|}
\hline Method & Advantages & Disadvantages \\
\hline $\begin{array}{l}\text { Van } \\
\text { (Mobile } \\
\text { Measurement } \\
\text { Unit) }\end{array}$ & $\begin{array}{l}\text { - Can collect at near highway speeds } \\
\text { - Flexibility in choosing technologies } \\
\text { - Can carry redundant systems } \\
\text { - High accuracy possible * }\end{array}$ & $\begin{array}{l}\text { - Technology not fully developed yet } \\
\text { - If buying, requires large investment } \\
\text { - May require several passes on a particular } \\
\text { road } \\
\text { - Skilled crew required* }\end{array}$ \\
\hline $\begin{array}{l}\text { Hand held } \\
\text { Measurement } \\
\text { Unit }\end{array}$ & $\begin{array}{l}\text { - Provides numerical data to compare } \\
\text { against proposed standards }\end{array}$ & $\begin{array}{l}\text { - Need to stop the vehicle and become aligned } \\
\text { with the sign } \\
\text { - Multiple measurements required for each sign } \\
\text { - Instrument accuracy uncertain }\end{array}$ \\
\hline $\begin{array}{c}\text { Visual } \\
\text { Inspection }\end{array}$ & $\begin{array}{l}\text { - Evaluation rate is fairly quick } \\
\text { - Trained crews available }\end{array}$ & $\begin{array}{l}\text { - No numerical data generated } \\
\text { - Labor and time intensive } \\
\text { - Does this method provide sufficient liability } \\
\text { protection? }\end{array}$ \\
\hline
\end{tabular}

* (Hummer, Karimi, and Khattak, 2000) 
road shoulder was established when using the hand-held retroreflectometer. Based on this measurement rate, it would require 42,500 person-hours to measure the North Carolina DOT's warning and stop signs.

\section{QUANTITATIVE EVALUATION OF THE VISUAL INSPECTION METHOD}

The previous sections revealed that using retroreflectometers to measure the traffic signs along the state-maintained roads in North Carolina, either using a hand-held unit or a mobile measurement unit (van), appears infeasible and impractical. The visual inspection method currently being used in North Carolina apparently should continue to be used to evaluate sign conditions.

However, the accuracy of the visual inspection method is questionable. As noted earlier, the human factor is critical when this method is used. Different observers will have different judgments about the condition of the sign being observed. Even the same observer might have different judgments on the same sign if it were evaluated several times during a short period when the sign condition had no significant changes. There is a need to evaluate this visual inspection method while taking into considerations factors such as the observers' accuracy in determining the signs' conditions, the inspection frequency, and vandalism.

This section describes a quantitative evaluation of the visual inspection method. The model is based on data from the Washington State Department of Transportation Traffic Sign Retroreflectivity Measurements Using Human Observers study (Lagergren, 1987).

\section{Washington State Study}

The Washington State study was based on 17 observers' ratings of warning and stop signs in a laboratory setting, a controlled highway setting, and an uncontrolled highway setting (Lagergren, 1987). Its purpose was to compare "the individual observer rating of the signs and the rating of the signs calculated by using retroreflectometer." Warning and stop signs were chosen because of their "high relative importance" and because they are commonly used on the roads. The uncontrolled highway setting was placed on two road types, a rural highway containing 76 signs and an urban highway containing 54 signs. Figures 1 and 2 represent the primary results of the uncontrolled highway portion of the study using the data based on the median results of 17 observers' ratings of 86 warning signs and 44 stop signs.

Sign sheeting type was not a factor during any portion of the study. This is congruent with North Carolina sign inspection practices because sign sheeting type is not considered during nighttime visual sign inspections, only whether the sign is sufficiently visible or not. The observers in the Washington State study rated the retroreflectivity of signs based on their visual judgments using a scale of 0 to 4 . Table 2 lists each rating category, the corresponding coefficient of retroreflection (RA), which is described as "specific intensity per unit area," or SIA, and a description of the category. Any signs rated 0 or 1 would be replaced and signs receiving a rating of 2,3 , or 4 would remain in place. Although the observers in the study received only limited amounts of training the

Table 2: Sign Ratings - Washington State Study

\begin{tabular}{|c|c|l|}
\hline Rating & $\begin{array}{c}\text { Corresponding SIA } \\
\text { Value (cd/sf/fc)* }\end{array}$ & \multicolumn{1}{c|}{ Description } \\
\hline 0 & 07 & Worst retroreflectivity \\
\hline 1 & 719 & Low retroreflectivity or other defect, sign ready for replacement \\
\hline 2 & 1937 & $\begin{array}{l}\text { Adequate retroreflectivity, looks okay, some defects but does not need } \\
\text { replacement }\end{array}$ \\
\hline 3 & 3770 & Good retroreflectivity \\
\hline 4 & $>70$ & Brand new sign \\
\hline
\end{tabular}

$* \mathrm{~cd} / \mathrm{sf} / \mathrm{fc}$ represents candelas per foot candle per square foot 
"inconsistency among observers was averaged in the median decision" (Lagergren, 1987).

In Figure 1, of the $74 \%$ reported accuracy for warning signs, $50 \%$ was the correct decision not to replace a sign (correct negative) and 24\% was the correct decision to replace a sign (correct positive). Of the $26 \%$ inaccuracy, $6 \%$ of the signs should have been replaced and were not (false negative) and $20 \%$ of the signs should not have been replaced and were (false positive). Thus the observers identified $44 \%$ of the signs as needing replacement $(20 \%+24 \%)$. Retroreflectometers identified $30 \%$ of the warning signs, whether evaluated correctly by observers or not, as needing replacement. Thus, the observers erred on the safer side (44\% vs. $30 \%$ ).

In Figure 2, of the $75 \%$ reported accuracy for stop signs, $32 \%$ was the correct decision not to replace a sign (correct negative) and $43 \%$ was the correct decision to replace a sign (correct positive). Of the $25 \%$ inaccuracy, $6 \%$ of the signs should have been replaced and were not (false negative) and 19\% of the signs should not have been replaced and were (false positive). In this case the observers identified $62 \%$ of the signs as needing replacement $(19 \%+43 \%)$. A total of $49 \%$ of stop signs needed replacement as identified by a retroreflectometer, whether evaluated correctly by observers or not. Thus, as with warning signs these figures show a conservative approach taken by the observer $(62 \%$ vs. $49 \%$ ).

Figures 3 and 4 show the frequency distributions of the observer ratings for the warning and stop signs of the Washington State Study. The X-axis represents the sign observers' ratings and the $\mathrm{Y}$-axis represents the sign ratings as determined by a retroreflectometer. Each cell

Figure 1: Decision Percentages for Warning Signs • Washington State Study

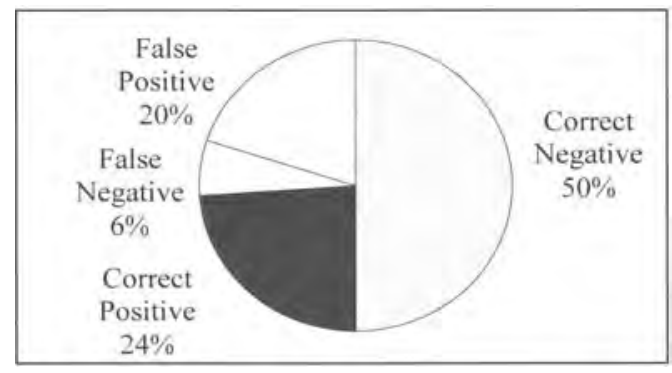

represents the number of signs and the percentage of signs corresponding to the sign observers' ratings and the sign ratings determined by a retroreflectometer. For example, cell $(0,0)$ indicates that of the 99 signs that were categorized into category 0 by the retroreflectometer, $87 \%$ were categorized into category 0 by the observers using the visual inspection method. The data in these two figures will be used in our analysis, described in the next section.

Figures 3 and 4 merit a few additional comments. The scale on the left is the SIA code indicating the retroreflectivity of the sign. SIA is correlated with sign age due to the fact that the signs toward the top of the scale are newer and those toward the bottom are older. The sign category $(0,1,2,3,4)$ indicates the rating the sign received by the inspector, with ratings defined in Table 2.

The small sample of observers in the Washington State Study limits the overall impact of the study's results. However, the Washington State Study was the only one available to use in our analysis.

\section{Methodology}

The methodology introduced here was developed to simulate the sign inspection and replacement process.The method has the following general assumptions:

- The inspectors in the agency of interest are as good as those in the Washington State study.

- In keeping with the fact that most signs installed on North Carolina public roads use engineer-grade sheeting, we assume that a sign has a useful life of seven years, after

Figure 2: Decision Percentages for Stop Signs • Washington State Study

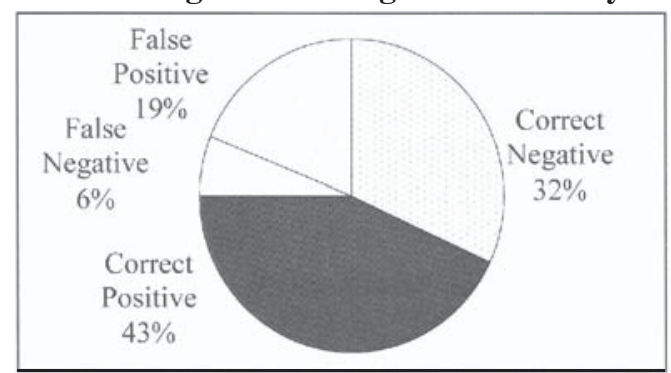


Figure 3: Frequency Distribution of Observer Ratings for Warning Signs Washington State Study

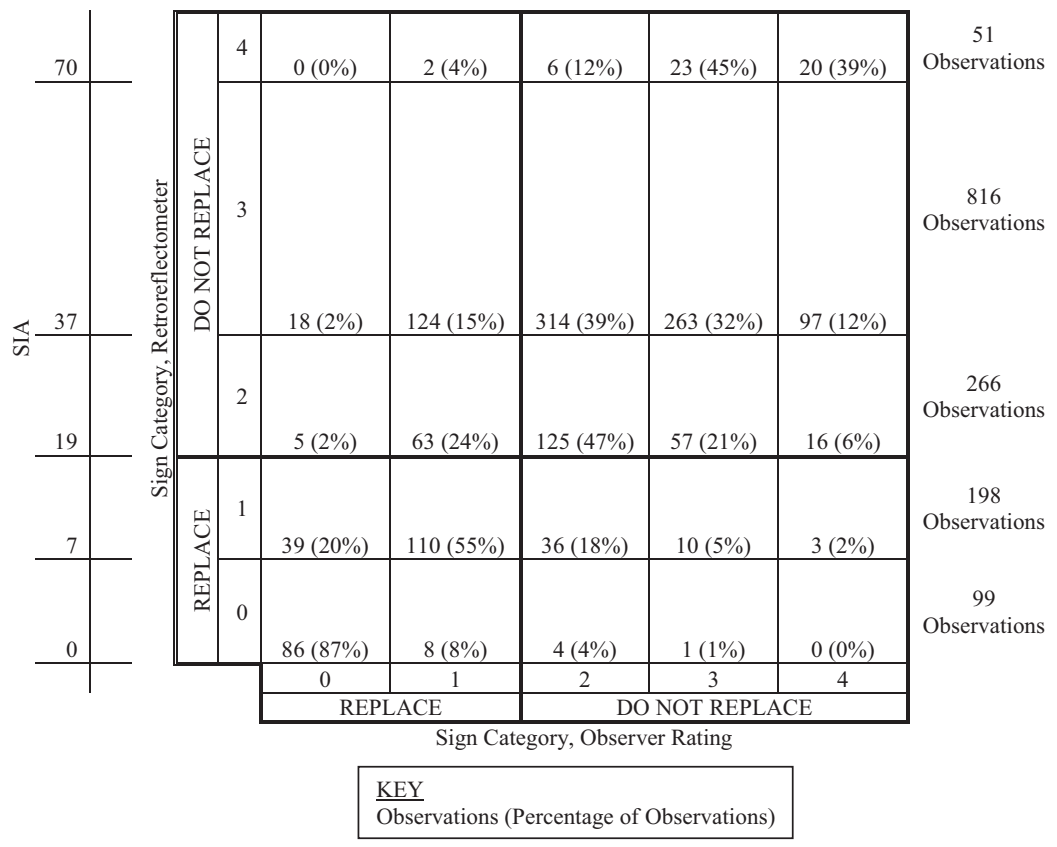

Figure 4: Frequency Distribution of Observer Ratings for Stop Signs Washington State Study

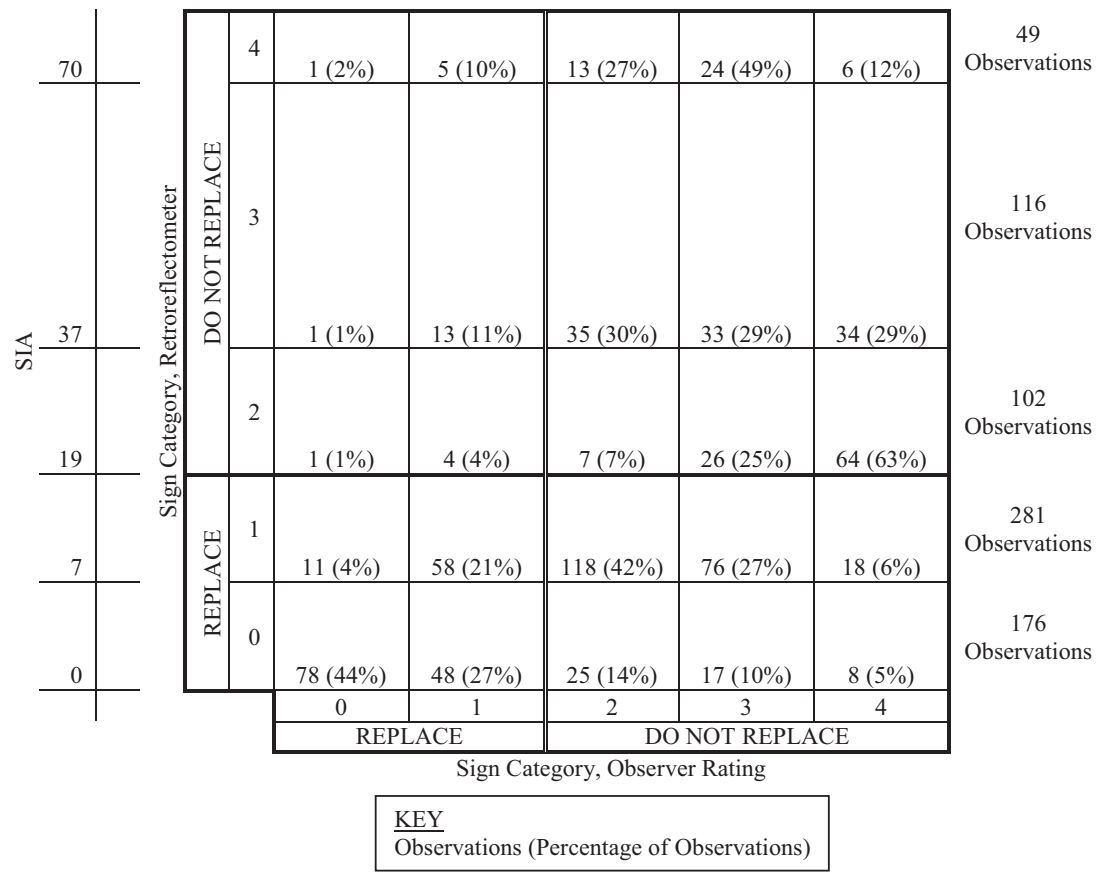


which it will need replacing. This assumption is neither a stated standard nor a written North Carolina DOT policy. However, it is an appropriate conservative estimate for engineer grade sheeting, and the results from our analysis do not change greatly with moderate changes in that useful life.

- The maximum SIA (RA) value of a new engineer grade sign is 70 (as cited in the Washington State study) and it was assumed to decrease by $1 / 7$ each year for seven years. Based on NCDOT data, this degradation assumption is very conservative considering that signs would most likely not degrade at a linear rate and would not be at an SIA value as low as 10 in year 7 . However, this conservative assumption also accounts for signs that may degrade faster than anticipated due to weather or damage.

- The assumed correspondence between sign age and SIA value is shown in the box below.

The analysis examined three key factors of an inspection program: inspection frequencies, replacement rates, and vandalism and knockdown rates. Three inspection frequencies were examined: each sign was inspected once a year, once every two years, or once every three years. These are frequencies currently used by the North Carolina DOT on various types of roadways. The replacement rates were determined based on the Washington State study: either inspectors would recommend sign replacement at the same rates as that study or more conservatively. Three different vandalism and knock-down rates were examined: no vandalism or knock downs, $5 \%$ of signs vandalized or knocked down per year, or $10 \%$ of signs vandalized or knocked-down per year. The North Carolina DOT believes that the $5 \%$ rate is appropriate for signs in urban areas while a $10 \%$ rate is appropriate for signs in rural areas. The different combinations of these factors were examined to determine their effects on the number of deficient signs (more than seven years old) that would still be in place on the road.
Replacement Rate. Our analysis required a replacement rate by sign age. Some of these rates were easy to discern from the Washington State study data, which was based on warning signs and stop signs. For example, based on Figure 3, for warning signs with roughly an SIA value of 10 (which we assume to be about six years old), the replacement rate is about $20 \%+55 \%=75 \%$. (Note that the value 10 does not explicitly appear on the scale, but it can be interpolated. It simply means that the sign is performing very poorly.) That is, the observer rated $20 \%$ of the signs as category 0 and $55 \%$ of the signs as category 1 meaning that they are to be replaced. Thus, the observer recommends replacing $75 \%$ of the signs that are six years old (with SIA of 7) but does not recommend replacing $25 \%(18 \%+5 \%+2 \%)$ of these signs. However, replacement rates for other sign ages were not as apparent from the data.

In the situation where the replacement rate could not be determined directly (for example, the replacement rate for warning signs with an SIA of approximately 50, which we assume to be two years old), two approaches were taken. The "typical rate" approach assumed that the replacement rate for signs at this specific age was the same as the replacement rate of the signs at the next-younger age for which there were available data. For example, the replacement rate for two-year-old warning signs would be the same as the replacement rate for new warning signs (SIA $=70)$, which is $4 \%$ according to upper left part of Figure $3(0 \%+4 \%)$. The "interpolation" approach used a linear interpolation from the replacement rates of signs at the next-younger and next-older ages for which there were data. For example, the replacement rate for one-year-old warnings signs $(\mathrm{SIA}=60)$ could be interpolated from the replacement rates of new $(\mathrm{SIA}=70)$ and three-year-old $(\mathrm{SIA}=37)$ signs as $(4 \%+0 \%)+$ $\{[(15 \%+2 \%)-(4 \%+0 \%)] / 3\}=8.33 \%$. That is, the replacement rate for new signs ( 0 years old) is $(4 \%+0 \%)=4 \%$ and the replacement rate for three-year-old signs (use the data for SIA=37) is $(15 \%+2 \%)=17 \%$. Interpolate one third of the

\begin{tabular}{|lllllllll|}
\hline Sign age, years: & 0 & 1 & 2 & 3 & 4 & 5 & 6 & $\geq 7$ \\
Corresponding SIA Value: & 70 & 60 & 50 & 40 & 30 & 20 & 10 & 0 \\
\hline
\end{tabular}


way from $4 \%$ to $17 \%$ to find the one-year-old sign replacement rate.

In addition, more conservative replacements were also examined. The "more conservative" replacement rates were based on the typical replacement rates, but assumed that inspectors were trained to look ahead several years and order sign replacement when they thought a sign would need to be replaced near the time of their next visit. More specifically, for the inspection frequency of once every year, the typical replacement rates were moved up one year to obtain the more conservative replacement rates. If the inspection frequency is once every two years, the typical replacement rates were moved up two years to obtain the more conservative replacement rates. If the inspection frequency is once every three years, the typical replacement rates were moved up three years to obtain the more conservative replacement rates. The more conservative replacement rates are illustrated in Table 3.

An example showing how these conservative replacement rates work follows. In Table 3 , the "typical" replacement rate for three-yearold signs is 0.17 (top row column 4). If we are instructing our inspectors to be conservative by one year they will reject two-year-old signs at the same rate as they "typically" rejected threeyear-old signs, 0.17 , as the third row (column 3 ) of Table 3 shows. If we are instructing our inspectors to be conservative by two years they will reject one-year-old signs at the same rate as they "typically" rejected three-year-old signs, 0.17 , as the fourth row (column 2) of Table 3 shows. If we are instructing our inspectors to be conservative by three years they will reject new signs at the same rate as they "typically" rejected three-year-old signs, 0.17 , as the fifth row (column 1) of Table 3 shows.

Simulation Procedure. To analyze the sign inspection program variables, we developed a simple simulation program in a spreadsheet. Once the program reaches stability, the result is a prediction of the number of deficient signs (seven or more years old) in the field. Table 4 illustrates the results of the simulation program assuming an even distribution of signs ranging from new to six years old as the initial starting state. The program application in this case was for warning signs and used an inspection frequency of once per year, the "more conservative" replacement rates, and a vandalism rate of $10 \%$ per year. Only the data from the first three years of the simulation are shown. It typically took about 30 years or so for the results to stabilize (be the same from year to year) and our results shown in Tables 5, 6, and 7 are for the end of a 60-year period.

In Table 4, each row represents the results at the end of that year's sign inspection cycle. Note that Table 4 is one continuous spreadsheet illustrated in 3 parts. The rows extend over a total of 56 columns from column A to BD. Column A with the heading of "Year" indicates the year the data represents. The first group of columns (B - J) under the heading of "In place signs" represents the sign age distribution at the end of that year. Except for the first year, the

Table 3: Sign Replacement Rates

\begin{tabular}{|c|c|c|c|c|c|c|c|c|c|c|}
\hline \multicolumn{3}{|c|}{ Sign Age (Years) } & 0 & 1 & 2 & 3 & 4 & 5 & 7 & $\geq 7$ \\
\hline \multirow{5}{*}{$\begin{array}{c}\text { Replacement } \\
\text { Rate (Warning } \\
\text { Signs) }\end{array}$} & \multirow{2}{*}{\multicolumn{2}{|c|}{$\begin{array}{l}\text { Typical } \\
\text { Interpolation }\end{array}$}} & 0.04 & 0.04 & 0.04 & 0.17 & 0.17 & 0.26 & 0.75 & 0.95 \\
\hline & & & 0.04 & 0.083 & 0.127 & 0.17 & 0.215 & 0.26 & 0.75 & 0.95 \\
\hline & \multirow{3}{*}{$\begin{array}{l}\text { More } \\
\text { Conservative* }\end{array}$} & 1 & 0.04 & 0.04 & 0.17 & 0.17 & 0.26 & 0.75 & 0.95 & 0.95 \\
\hline & & $1 / 2$ & 0.04 & 0.17 & 0.17 & 0.26 & 0.75 & 0.95 & 0.95 & 0.95 \\
\hline & & $1 / 3$ & 0.17 & 0.17 & 0.26 & 0.75 & 0.95 & 0.95 & 0.95 & 0.95 \\
\hline \multirow{5}{*}{$\begin{array}{c}\text { Replacement } \\
\text { Rate (Stop } \\
\text { Signs) }\end{array}$} & \multicolumn{2}{|l|}{ Typical } & 0.12 & 0.12 & 0.12 & 0.12 & 0.12 & 0.05 & 0.25 & 0.71 \\
\hline & \multicolumn{2}{|l|}{ Interpolation } & 0.12 & 0.12 & 0.12 & 0.12 & 0.085 & 0.05 & 0.25 & 0.71 \\
\hline & \multirow{3}{*}{$\begin{array}{l}\text { More } \\
\text { Conservative* }\end{array}$} & 1 & 0.12 & 0.12 & 0.12 & 0.12 & 0.05 & 0.25 & 0.71 & 0.71 \\
\hline & & $1 / 2$ & 0.12 & 0.12 & 0.12 & 0.05 & 0.25 & 0.71 & 0.71 & 0.71 \\
\hline & & $1 / 3$ & 0.12 & 0.12 & 0.05 & 0.25 & 0.71 & 0.71 & 0.71 & 0.71 \\
\hline
\end{tabular}

* The "more conservative" replacement rates are dependent on the inspection frequency as illustrated.

$1 / 2$ means signs inspected every other year

1.3 means signs inspected every third year 
values in this group of columns depend on the inspection, replacement, and vandalism activities in the previous year. For example, the first row indicates that by the end of year 1 , there are 143 new signs, 143 one-year-old signs, etc., according to the assumption of a uniform age distribution to begin the simulation. The second row indicates the sign age distribution by the end of year 2. By the end of year 2, the number of new signs is 406 , which equals the total number of signs replaced due to the year 1 inspection procedure $(6+6+24+24+37+107+136+0$ $=340$ signs) plus the number of signs replaced due to vandalism in the first year (66 signs). By the end of year 2, the number of one-year old signs is 123 , which equals the total number of the new signs that were either not inspected (0 signs) or not replaced (137 signs) in year 1 minus the number of new signs vandalized in year 1 (14 signs).

The second group of columns (K - S) under the heading of "Inspected signs" represents the number of signs of different ages being inspected during the inspection procedure, which was assumed to happen at the end of year 1 . The values in these columns are dependent on the number of in-place signs and the inspection frequency. For example, there are 143 new signs in year 1 and the inspection frequency is once per year. In other words, all these 143 new signs will be inspected and therefore, the number of inspected new signs is 143 .

The third group of columns (U - AC) under the heading of "Not Inspected signs" represents the number of signs that are not inspected in that specific year. The values are obtained by subtracting the number of signs inspected in that year from the number of in-place signs in that year grouped by the sign age. Since the inspection frequency is once per year in this example, all signs will be inspected and there are zeroes in all cells of this group of columns.

The fourth group of columns (AD - AK) under the heading of "Replaced signs" represents the number of signs that are replaced due to the inspection and replacement procedure in that specific year, grouped by age. The values depend on the number of signs and the corresponding replacement rate. For example, for a new sign, the replacement rate is 0.04 and there are 143 new signs in year 1 . Therefore, six $(143 * 0.04)$ new signs were replaced due to the inspection and replacement procedure in year 1 .

The fifth group of columns (AM - AT) under the heading of "Not replaced signs" represents the number of signs that are not replaced as a result of that year's inspection and replacement procedure. The values are obtained by subtracting the number of "Replaced signs" from the number of "In place signs" of the corresponding sign ages.

Column AU, under the heading of "Not inspected or not replaced," represents the total number of signs that are either not inspected or not replaced during the specific year's inspection and replacement procedure.

The final group of columns (AV - BD) under the heading of "Vandalized signs" represents the number of signs replaced due to vandalism, knockdown, or similar causes unrelated to the sign inspection program, from among those signs that were "Not inspected or not replaced," again grouped by sign age. The values are obtained by applying the vandalism rate to the corresponding "Not inspected or not replaced" signs. For example, in year 1 there are 137 new signs that are either not inspected (0) or not replaced (137) during the sign inspection and replacement procedure. With a vandalism rate of $10 \%$, the number of vandalized new signs is 14 after rounding up $(137 * 10 \%=13.7)$.

Note that with a $0 \%$ vandalism rate, the number of new signs in year $\mathrm{n}$ is the number of signs replaced due to the year $n-1$ sign inspection and replacement procedure. If the vandalism rate is greater than $0 \%$, the number of new signs in year $\mathrm{n}$ includes the number of signs replaced due to the year n-1 inspection and replacement procedure and the signs vandalized in year $\mathrm{n}-1$. The simulation logic is provided mathematically below for those readers interested in replicating the calculation.

- Given the number of years between inspections, ybi;

- Given the proportion of signs vandalized in a year, pv;

- Given the proportion of signs of a given age, a, that inspectors will recommend to 

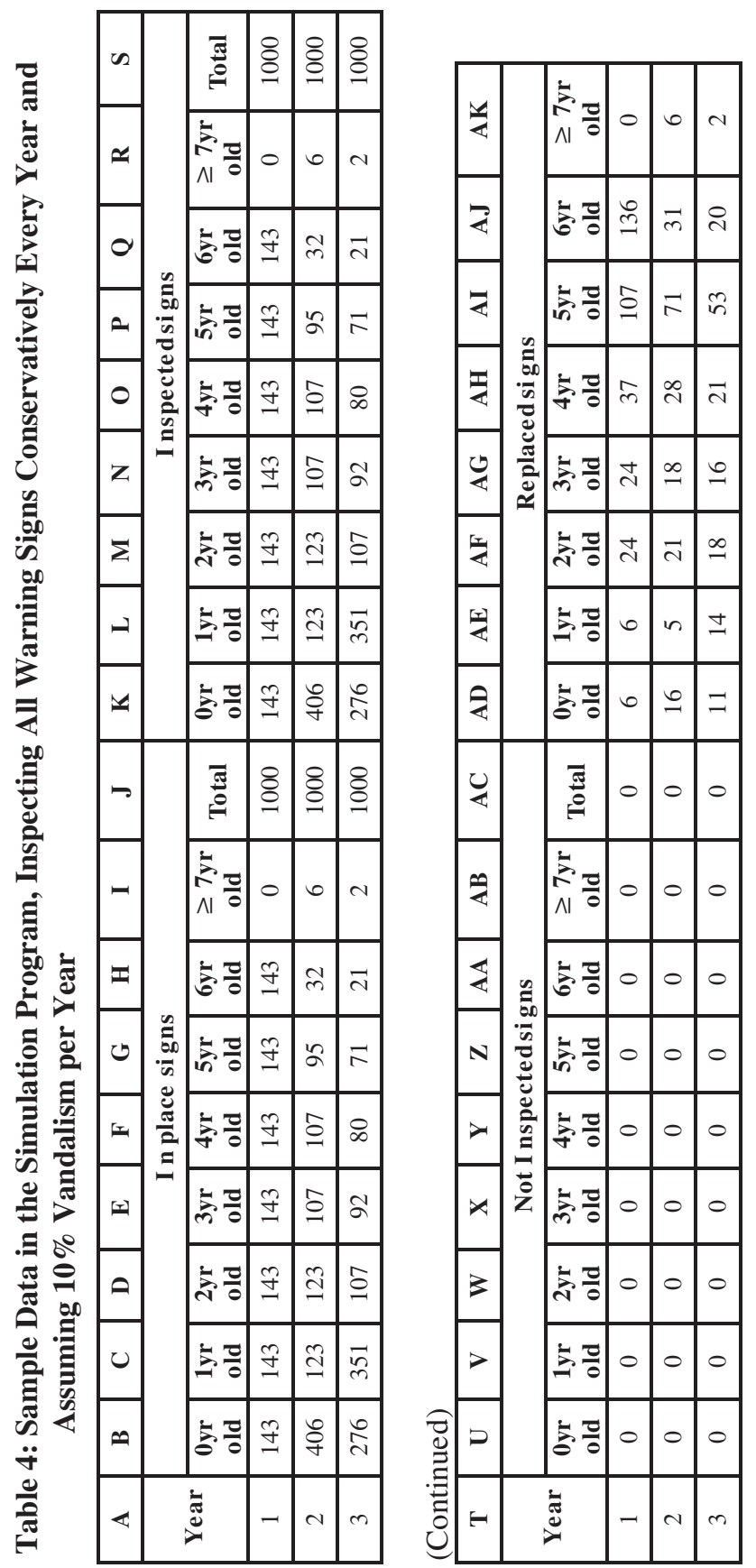

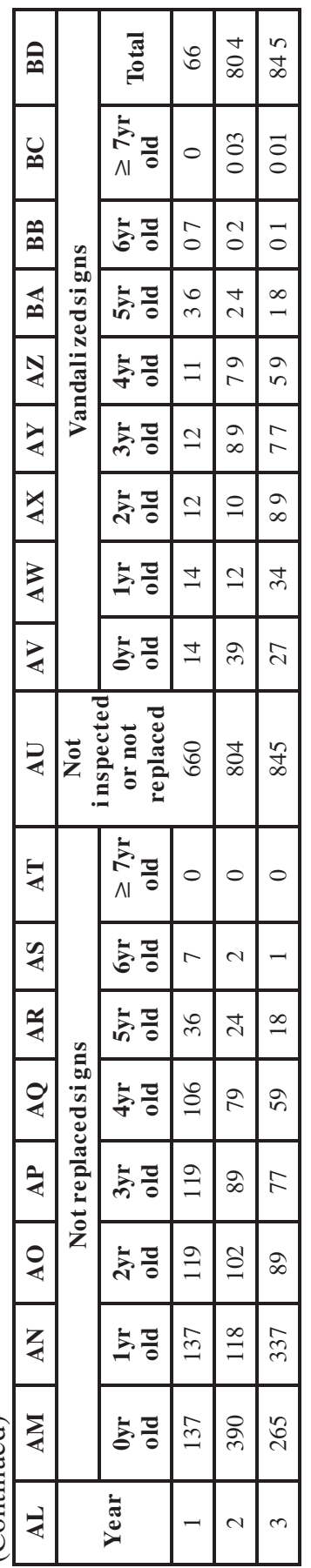


be replaced, $\mathrm{RR}_{\mathrm{a}}$ (a "replacement rate" from Table 3); and

- Given a beginning distribution of in-place signs, $\mathrm{P}$, in year $\mathrm{y}$, by age, or a set of $\mathrm{P}_{\mathrm{y}, \mathrm{a}}$ :

1. Compute the number of signs of age a to be inspected in year $\mathrm{y}, \mathrm{I}_{\mathrm{y}, \mathrm{a}}=\mathrm{P}_{\mathrm{y}, \mathrm{a}} / \mathrm{ybi}$;

2. Compute the number of signs of age a not to be inspected in year $\mathrm{y}, \mathrm{NI}_{\mathrm{y}, \mathrm{a}}=\mathrm{P}_{\mathrm{y}, \mathrm{a}}-\mathrm{I}_{\mathrm{y}, \mathrm{a}}$;

3. Compute the number of signs of age a to be replaced in year $y, \mathrm{R}_{\mathrm{y}, \mathrm{a}}=\mathrm{I}_{\mathrm{y}, \mathrm{a}} * \mathrm{RR}_{\mathrm{a}}$;

4. Compute the number of signs of age a not to be replaced in year $y, \mathrm{NR}_{\mathrm{y}, \mathrm{a}}=\mathrm{P}_{\mathrm{y}, \mathrm{a}}-\mathrm{R}_{\mathrm{y}, \mathrm{a}}$;

5. Compute the number of signs of age a vandalized in year $\mathrm{y}, \mathrm{V}_{\mathrm{y}, \mathrm{a}}=\mathrm{NR}_{\mathrm{y}, \mathrm{a}} * \mathrm{pv}$;

6. Compute the number of new signs in year $\mathrm{y}+1, \mathrm{P}_{\mathrm{y}+1,0}$

$$
=\stackrel{y+1,0}{=} R_{y, a}+\sum V_{y, a} ;
$$

7. Compute the number of signs of ages one year and older, $\mathrm{P}_{\mathrm{y}+1, \mathrm{a}}($ where $\mathrm{a} \geq 1)$

$$
=\mathrm{NI}_{\mathrm{y}, \mathrm{a}-1}+\mathrm{NR}_{\mathrm{y}, \mathrm{a}-1}^{\mathrm{y}+1, \mathrm{a}}-\mathrm{V}_{\mathrm{y}, \mathrm{a}-1} \text {; and }
$$

8. Return to Step 1 and repeat the computation for the next year, $y+1$. Keep repeating the computation until the simulation stabilizes, which is to say that $\mathrm{P}_{\mathrm{y}, \mathrm{a}} \approx \mathrm{P}_{\mathrm{y}+1, \mathrm{a}}$

\section{SIMULATION RESULTS}

After the simulation program was developed, several different combinations of the replacement rates, inspection frequencies, and vandalism rates were run in the program for both warning signs and stop signs. All cases assumed a sign sample of 1,000 and, as noted above, started with a uniform distribution of sign ages, although that did not matter in the final results since we simulated a long enough time period for the system to stabilize.

The simulation program was applied to the hand-held retroreflectometer method for comparison and evaluation purposes. When using a hand-held retroreflectometer to measure the retroreflectivity of a sign, an almost perfect performance was assumed. In other words, it was assumed that the retroreflectometer could provide the operator with enough information to make a correct decision on replacement $99 \%$ of the time. The decision to replace a sign or not was made based on the sign age and the inspection frequency. For example, if the inspection frequency is once every two years, signs at ages of five years, six years, and seven years or older would be replaced $99 \%$ of the time. Signs at age of five years would be replaced even though these signs could last another year to make sure they would not stay in field while running out of their useful life before the next inspection.

To round out the field of contending inspection and replacement strategies, we analyzed a "total replacement" approach as well. This idealized situation assumed no inspection; each sign was simply replaced in the seventh year since the previous mass installation.

Table 5 provides simulation results for warning signs and Table 6 provides results for stop signs. The simulation results are useful to evaluate the various sign inspection and replacement options. The focus is on the safety risk and the cost associated with each inspection program. The safety risk is dependent on the number of grossly deficient signs in place on the roads (assumed to be signs seven years or older in this study). Generally, the more of these signs there are in the field, the higher the safety risk. The cost includes the sign replacement cost and the sign inspection cost. The cost information used in this study is from sign inventory research sponsored by the North Carolina DOT (Vereen, Hummer, and Rasdorf, 2002). The sign replacement cost for warning and stop signs is $\$ 30 /$ sign. The sign inspection cost of the visual inspection method is $\$ 0.17 /$ sign based on the previous research. The inspection cost for the hand-held retroreflectometer method is estimated to be $\$ 2.33 /$ sign when taking into consideration the labor costs (including salaries and benefits), travel costs, vehicle costs, and equipment cost (retroreflectometer). Table 7 summarizes the cost and the safety risk associated with each inspection method examined for warning signs. 
Nighttime Visual Sign Inspection

\section{Observations}

Tables 5-7 provide several useful insights. Under the same conditions (inspection method, vandalism rate, replacement rate), the higher the inspection frequency, the higher the number of signs replaced every year, and the lower the number of signs that do not meet the requirements. In other words, the higher the inspection frequency, the higher the cost to inspect and replace signs, but the lower the safety risk. In addition, it is noted that with typical or interpolated visual inspection methods, the number of deficient signs is very high, and may be unacceptable, with every other year or every third year inspection.

Keeping the vandalism rate constant, the number of signs that do not meet requirements could be decreased somewhat using the handheld retroreflectometer method rather than the visual inspection method, but with a significant increase in the total cost. For example, under the vandalism rate of $10 \%$, with an inspection frequency of once every year, the cost for the visual inspection method using the typical replacement rate (item 27 in Table 7) is $\$ 7,010.00$ per year, which is $\$ 1,230.00$ (18\%) less than the cost of the hand-held retroreflectometer method ( $\$ 8,240.00$, from item 36 in Table 7$)$ for 1,000 warning signs. The estimated number of warning signs on NC roads is 373,000 , which leads to an estimated total cost difference of $\$ 458,790$ per year $((8,240.00-7,010.00) *$ $373)$. The number of signs that do not meet requirements is decreased from 13 out of 1,000 for visual inspection to one out of 1,000 for the retroreflectometer. Thus, there is very little improvement in safety risk ( $1.2 \%$ fewer grossly deficient signs) for a lot more cost.

For all cases, the majority of the cost comes from the replacement cost because the unit replacement cost is \$30/sign while the unit cost of inspection is only $\$ 0.17 /$ sign for the visual inspection method and $\$ 2.33 /$ sign for the hand-held retroreflectometer method. It is also obvious that the difference in inspection costs is important. Using the same example as in the previous paragraph, item 33 in Table 7 shows that the more conservative visual inspection method calls for replacement of many more signs than the retroreflectometer (item 36) to produce the same number of deficient signs (one per 1,000$)$. However, the greater inspection costs for the retroreflectometer give it a higher overall cost than the more conservative visual inspection method. Ways to make retroreflectometer use faster without sacrificing reliability would be helpful to the DOTs.

With the assumptions that were made, the total replacement approach turns out to be attractive when taking into consideration the cost and the number of deficient signs. When using the total replacement approach, the number of signs that do not meet the requirements is always 0 , and the total program costs are among the lowest we analyzed. However, in the real world where signs are not automatically deficient after seven years and DOTs cannot count on finding vandalized and knocked-down signs without regular inspections, the total replacement approach may not be realistic.

\section{CONCLUSION}

Organizations might have different goals in sign inventory and management. For example, some organizations might seek methods to minimize the cost while tolerating a relatively large number of signs that do not meet the retroreflectivity requirements. Some organizations might seek methods to minimize the number of signs that do not meet retroreflectivity requirements while tolerating a relatively high cost. Still other organizations might seek methods to obtain a balance, i.e., both the number of signs that do not meet the requirements and the cost are reasonable. The simple spreadsheet simulation program described in this paper appears to be a way for agencies to explore the trade-offs and arrive at informed decisions on their sign inspection and replacement efforts.

For agencies that generally conform to the key assumptions made to build the spreadsheet (such as uniformly decaying signs that become deficient after seven years in the field, inspectors with error rates similar to the Washington State study (Lagergren, 1987), and virtually flawless inspections with retroreflectometers), the results in Tables 5-7 are directly applicable. Those agencies should consider replacing all 
signs every seven years, as that insures that at a relatively low cost no aged signs are in place, subject to the key realism checks mentioned above. If total replacement is not possible, an inspection program using retroreflectometers every three years appears very cost competitive with a program using "typical" visual inspection rates each year at either $5 \%$ or $10 \%$ vandalism rates. The retroreflectometers appear to allow fewer deficient signs, while the "typical" visual inspection program costs are lower for a given vandalism rate. "More conservative" sign replacement rates do not appear to offer distinct advantages, while "typical" replacement rates with inspections every two or three years allow relatively high numbers of deficient signs to remain on the roads.

For other agencies, they can easily change the parameters of the simulation program to different values than those used in this study. For example, if their documented vandalism rate differs from the one we used they can simply change that one parameter and retain the use of the program to generate new values for sign replacement rates. Among the parameters that can be changed to allow a wider range of applicability of the method are the following:

- Replacement of the simple linear sign deterioration function by age with a more sophisticated function,

- Changing the overall duration of the determination cycle,

- Addition of other grades of sheeting,

- Addition of data on inspector performance from other DOTs,

- Addition of other sign colors, and

- Addition of data on the error rates from retroreflectometers.

The authors plan to pursue research along several of these lines in the near future. As more of these parameters are validated for individual uses over time the sign inspection and replacement simulation program will become an increasingly powerful tool for helping DOTs save lives and money. 


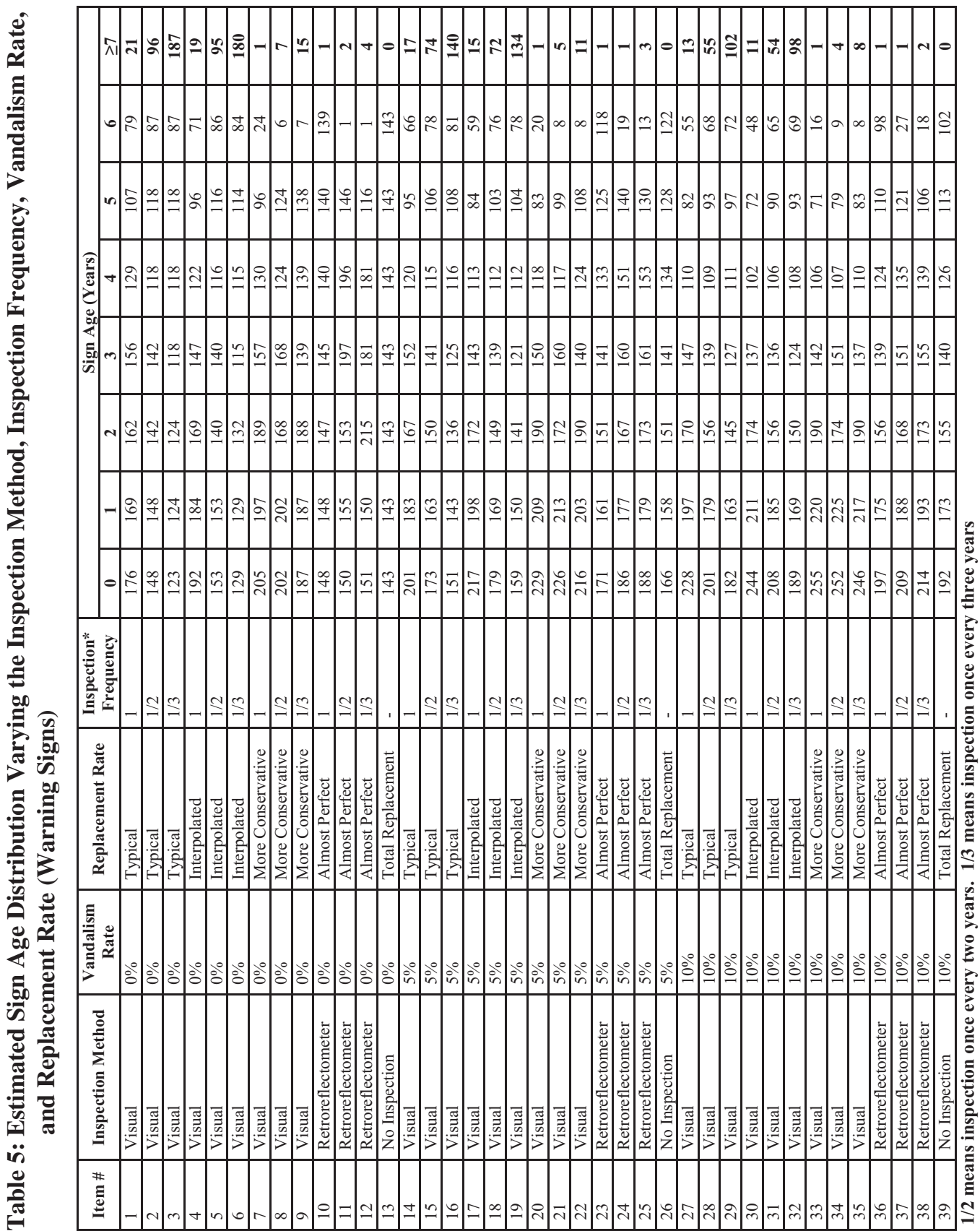




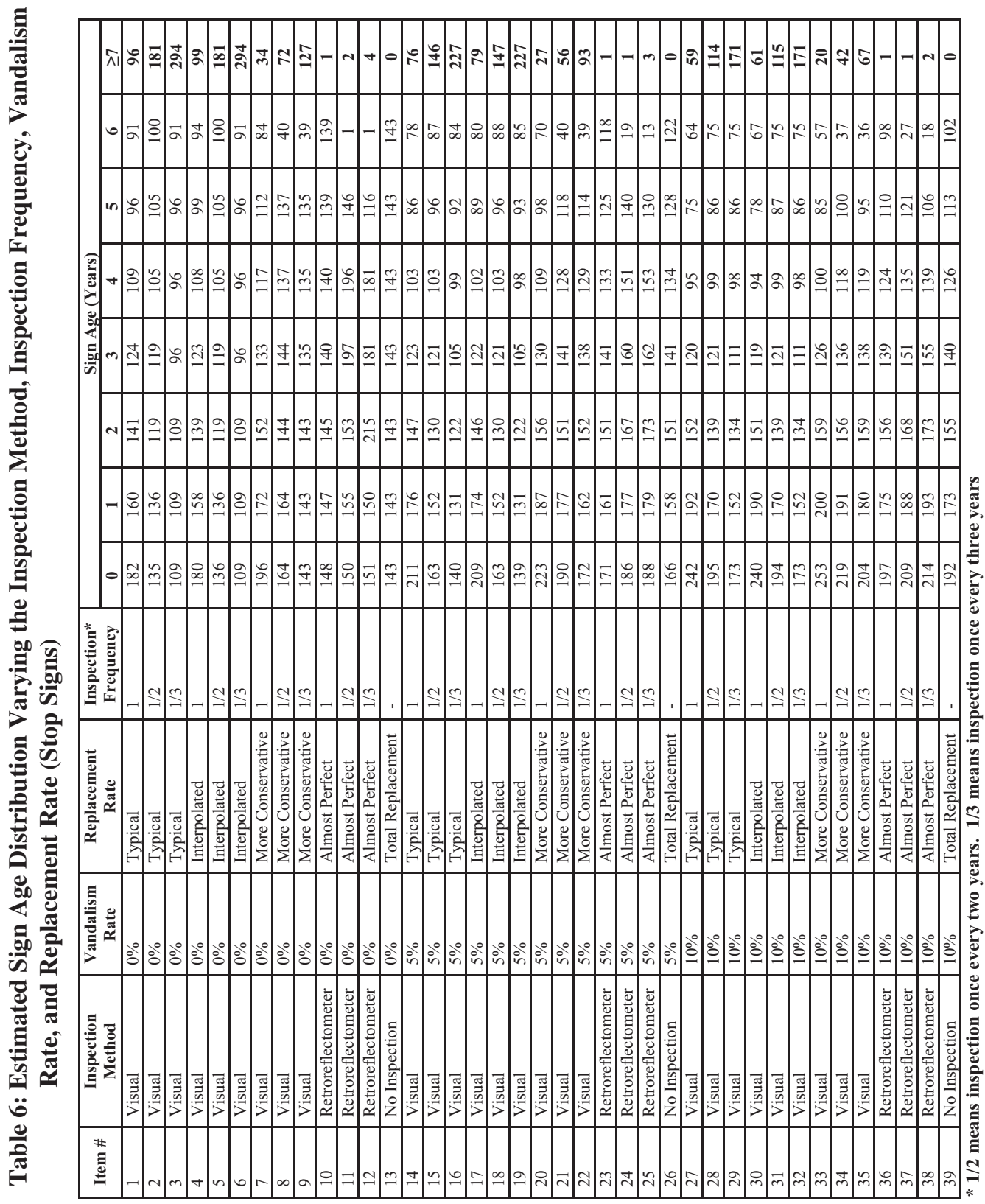




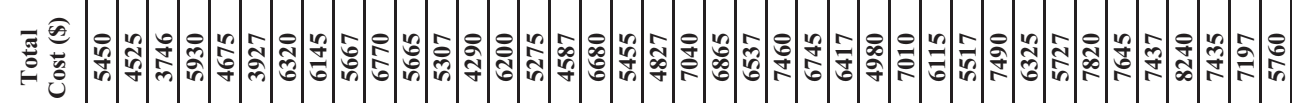

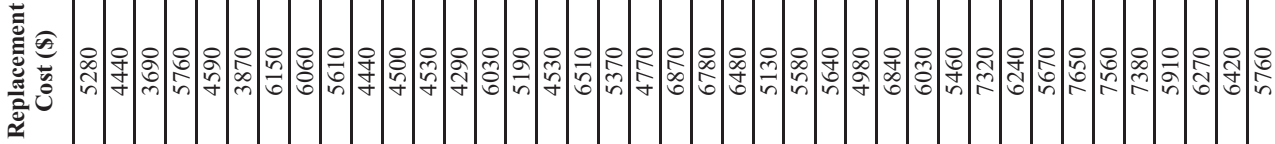

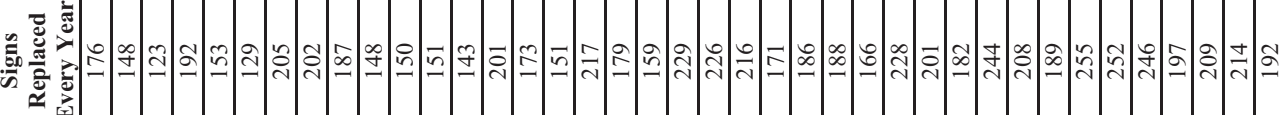

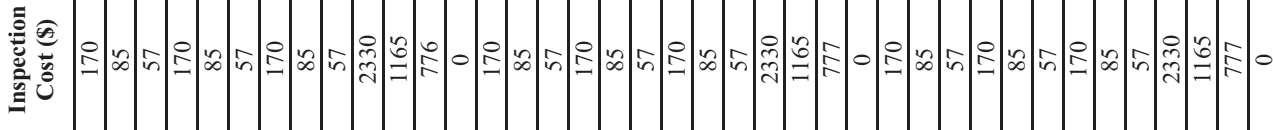
空

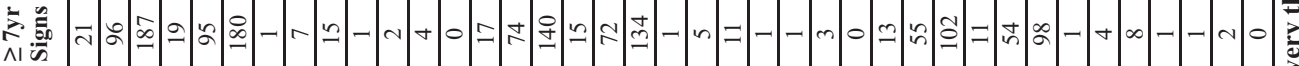

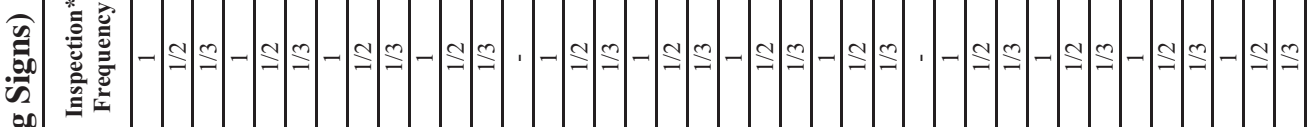

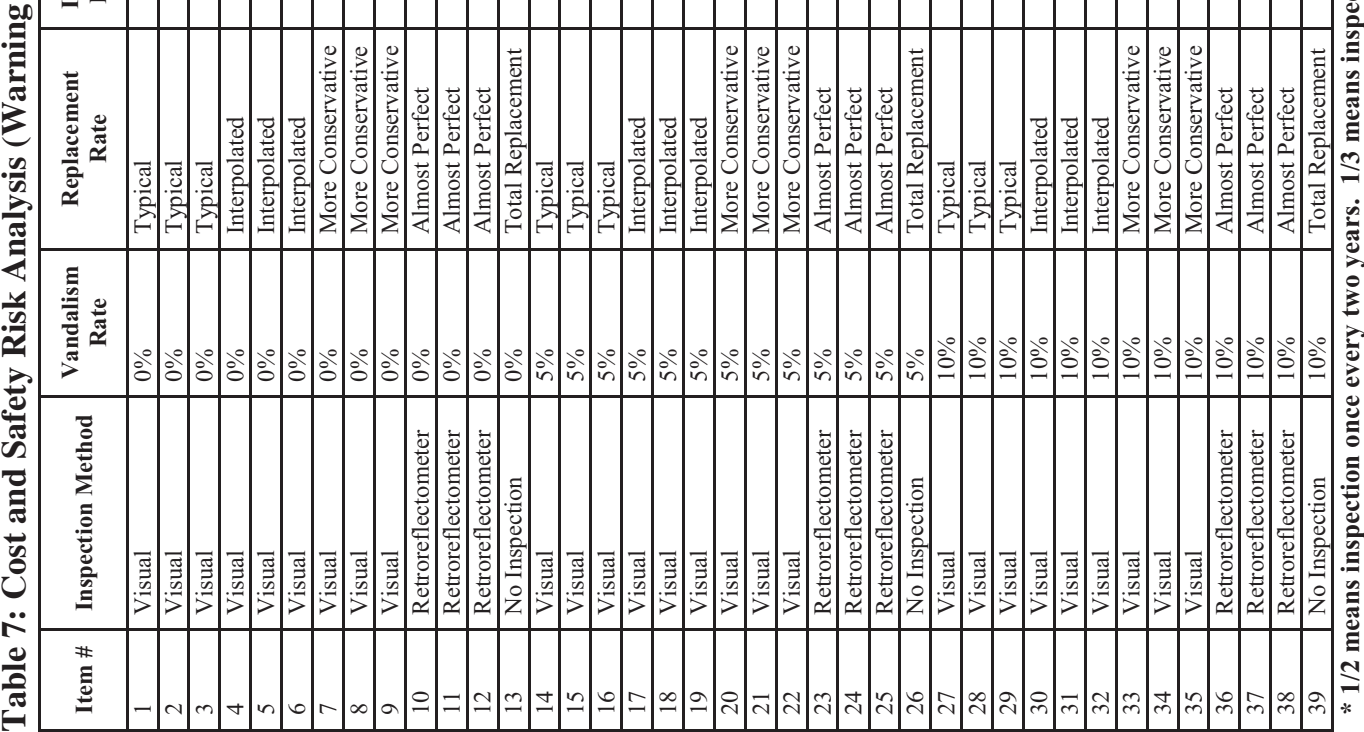




\section{References}

AASHTO, Retroreflectivity Policy Resolution, Minimum Levels of Retroreflectivity for Signs, December 9, 2000.

Congressional Information Service, Inc. "Crashes By Relation to Junction, Traffic Control Devices, and Crash Severity." Traffic Safety Facts, 1999.

Hasson, P. Retroreflectivity: An Essential Tool for Improving Visibility. http://mrc.fhwa.dot.gov/ articles/ksart2.htm, U.S. Department of Transportation, Federal Highway Administration, Midwest Resource Center, 1999.

Hatzi, P., "Retroreflectivity 'Right Back At You!” U.S. Department of Transportation, Federal Highway Admin., http://www.library.unt.edu/gpo/OTA/featproj/fp_te29.html, June 30, 2001.

Hummer, J., A. H. Karimi, and A. Khattak. Collection and Presentation of Roadway and Inventory Data. NCHRP Report 437, Transportation Research Board, National Academy Press, Washington, D.C., 2000.

Institute of Transportation Engineers. Traffic Signing Handbook, Institute of Transportation Engineers, Washington, D.C., Chapter 2, (1997): 23 - 44.

Kirtley, N. and W. Rasdorf. North Carolina Sign Count Study \#1 Primary Roads. North Carolina State University, 2001.

Lagergren, A. E. Traffic Sign Retroreflectivity Measurements Using Human Observers. Washington State Department of Transportation, WA-RD 140.1, December, 1987.

Long, D. "Michigan DOT Reflects on Signs.” TR News, 192, September/October (1997): 24 - 25.

McGee, W. H. and A. J. Paniati. An Implementation Guide for Minimum Retroreflectivity Requirements for Traffic Signs. USDOT, FHWA Research and Development, McLean, VA, 1998.

McGee, W. H. and S. Taori. "Impacts on State and Local Agencies for Maintaining Traffic Signs Within Minimum Retroreflectivity Guidelines.” BMI, Vienna, VA, 1998.

National Cooperative Highway Research Program (NCHRP), Project 5-16. National Calibration Standards for Measuring Retroreflectivity, http://www4.nationalacademies.org/, last modified April 19, 2000, accessed May, 2003.

Palmquist, M. and W. Rasdorf. North Carolina Sign Count Study \#2 Secondary Roads. North Carolina State University, Raleigh, NC, 2002.

Vereen, C. S., E. J. Hummer, and W. J. Rasdorf. “A Sign Inventory Study to Assess and Control Liability and Cost." Final Report for NCDOT Research Project 2001-16, 2002. 


\section{Acknowledgements}

The project on which this paper was based was sponsored by the North Carolina DOT. The authors thank the North Carolina DOT for the support. The authors also thank the members of the project technical committee, the research and development staff, and the staff in many units who provided data, advice, and assistance of various kinds. The authors assume full responsibility for the accuracy of the data and conclusions presented in the paper.

William Rasdorf, P.E., is a Professor in the Department of Civil, Construction, and Environmental Engineering at North Carolina State University. Rasdorf has been on the faculty at NC State since 1982. He received B.S. and M.S. degrees in architectural engineering from Penn State University and M.S. and Ph.D. degrees in Civil Engineering from Carnegie Mellon University. Rasdorf specializes in computer applications in structural, construction, and transportation engineering as well as in manufacturing. Some of his current work focuses on transportation spatial and inventory data using an integrated GIS/database approach. Rasdorf has played a central role in the design of the base linear reference system (LRS) currently being developed by NCDOT for statewide use in both GIS and database environments. He is chairman of the ASCE Technical Council on Computer Practices' Publications Committee, and is the editor of ASCE'S Journal of Computing in Civil Engineering.

Joseph E. Hummer, P.E., is a professor in the Department of Civil, Construction, and Environmental Engineering at North Carolina State University. Hummer has been on the faculty at NC State since 1992. He previously taught at UNC Charlotte and was a graduate research fellow at the Federal Highway Administration. He received B.S. and M.S. degrees in civil engineering from Michigan State University and a Ph.D. in civil engineering from Purdue University. Hummer specializes in traffic operations, highway safety, and highway design. He has published over 100 papers and has participated in over 30 research projects for a variety of sponsors. In the area of signing, Hummer has investigated sign inventory systems; has examined the safety impacts of fluorescent yellowgreen, orange, and yellow signs; and has looked for new ways to convey information on left turns and horizontal curves.

Stephanie Vereen received her B.S. and M.S. degrees from NCSU in 2000 and 2002 respectively. She is currently a project engineer with CDM Constructors Inc. in Maitland, Fla., working on an $\$ 18.9$ million upgrade to an existing water treatment plant facility in Valdosta, Ga. She is responsible for coordinating submittals and for fabrication and delivery for owner purchased equipment. She coordinates the equipment and subcontractor bid periods and work tasks involved with job start-up (site set-up, aerial photos, pre-construction video, etc.). She also assists with resolving warranty items on equipment already installed and in use on a previous water reclamation facility project. Vereen currently holds an engineer intern license and achieved the passing requirements for International Code Council (ICC) Commercial Building Contractor B.

Hubo Cai came to the United States from China in 1999. From August 1999 to May 2000, Cai was a graduate student at Iowa State University majoring in construction engineering and management. He transferred to North Carolina State University in 2000. In 2001 Cai was awarded the degree of Master of Civil Engineering with a research focus on GIS in civil and transportation engineering. In December of 2003 he received his Ph.D. with a research focus on 3D spatial modeling and GIS applications in engineering from NC State. Upon graduation, Cai served as a GIS Specialist with the GIS Unit of the NC Department of Transportation (NCDOT) working on projects of Web-based GIS applications, spatial database development and management, and customized GIS tool development and providing support to other units within NCDOT. In June of 2004, Cai began work as a GIS analyst and GIS applications developer at URS Corp. - North Carolina. Presently he is working on several storm water management projects and GIS applications development projects. 